AUSTRALIAN JOURNAL OF BASIC AND
APPLIED SCIENCES
EISSN: 2309-8414
DOI: 10.22587/ajbas.2017.11.13.18
Journal home page: www.ajbasweb.com

\title{
A Comprehensive Review of the Confirmed Therapeutic Effects of Grape Seed Extracts, Cranberry Juice, and Proanthocyanidins in Human Beings
}

\author{
Arwa M. Turkistani \\ Department of Food and Nutrition, King Abdul-Aziz University, Jeddah, Saudi Arabia
}

Address For Correspondence:

Arwa M. Turkistani Department of Food and Nutrition, King Abdul-Aziz University, Jeddah, Saudi Arabia

\section{A RTICLE INFO}

Article history:

Received 15 October 2017

Accepted 18 November 2017

Available online 14 December 2017

Keywords:

Proanthocyanidins, therapeutic effects,

human beings, review.

\begin{abstract}
A B S T R A C T
Currently, grape seed extracts and cranberry juice are utilized in conventional medicine for their pharmacological attributes. Depending on fresh vegetables and fruits to amend human health is assigned mostly to their active constituents. Proanthocyanidins are the major components of grape seed extracts and cranberry juice which are also present in other plants kind. Proanthocyanidins exert numerous pharmacological actions comprising antimicrobial, anti-adhesion, antioxidant, and anti-inflammatory actions. They were established in berries especially, lingonberry, cranberry, black elderberry, black chokeberry, black currant, and blueberry. Lately, several trials evidenced the efficacy of grape seed extracts, cranberry juice, and proanthocyanidins in either treatment and/or prevention of dry skin problems, urinary tract infections, gingivitis, wound healing, leg swelling, radiation injury, Helicobacter pylori, and melasma. The therapeutic impacts of grape seed extracts, cranberry juice, and proanthocyanidins could be referred to as the clinically proved antioxidant, antiadhesive, and antiinflammatory activities. In conclusion, grape seed extracts, cranberry juice, and proanthocyanidins may be utilized as a treatment or adjuvant treatment to manage plentiful of diseases.
\end{abstract}

\section{INTRODUCTION}

Worldwide, there is increasing care in alternative therapy which is concomitant with the enormous amount of research works examining the pharmacological effects of the active components and their ability to cure various diseases (Yuan et al., 2016). Almost all drugs have entered the international market via examination of ethnopharmacology and traditional medicine. In spite of the huge number of plants and its active constituents that undergo research trials, but few numbers of medicines have been entered the market, and organic drugs have been incorporated in the evidence-based medicines (Pan et al ., 2013).

Flavonoids are a category of a polyphenolic substances that possesses beneficial health effects (Ravishankar et al., 2013). Several flavonoids like catechin and epicatechin polymerize to produce tannins. Tannins are secondary herbal metabolites that are either hydrolyzable or condensed. Generally, condensed tannins are recognized as proanthocyanidins (De la Iglesia et al., 2010). Proanthocyanidins have existed in flowers, nuts, fruits, bark, and seeds of several plants. They protect plants versus biotic and abiotic stimuli.

Furthermore, they conserve the herbs from pathogenic invasion. Proanthocyanidins are oligomeric and polymeric compounds obtained from the flavonoid biosynthetic pathway. The basic components of proanthocyanidins comprise catechin and epicatechin (Dong et al ., 2014).

Proanthocyanidins are found in large amount in berries especially, lingonberry, cranberry, black elderberry, black chokeberry, black currant and blueberry (Krenn et al., 2007). The A-linked proanthocyanidins are present in excess levels in cranberry, peanut skins and cinnamon (Singh et al., 2009 and Foo et al., 2000). The cranberry A-type proanthocyanidins are fundamentally formed of epicatechin monomer linked with

Open Access Journal

Published BY AENSI Publication

(C) 2017 AENSI Publisher All rights reserved

This work is licensed under the Creative Commons Attribution International License (CC BY).

http://creativecommons.org/licenses/by/4.0/

\section{(c) (i) Open Access}

To Cite This Article: Arwa M. Turkistani, A comprehensive review of the confirmed therapeutic effects of grape seed extracts, cranberry juice, and proanthocyanidins in human beings $\mathrm{A}^{2}$ Aust. J. Basic \& Appl. Sci., 11(xx): xx-xx, 2017 
minimally one A-type interflavan bond. The type of A-type interflavan linkage is tightly associated with its biological activities. The A-type proanthocyanidins evidenced to be better bioactive than B-type proanthocyanidins (Singh et al., 2009 and Howell et al., 2005). For example, A-type proanthocyanidins showed significant anti-adhesion impact versus $E$. coli and $H$. pylori, while B-type proanthocyanidins were not (Nicolosi et al., 2014). Cranberry A- proanthocyanidins has been proposed to significantly decrease the occurrence and intensity of smooth surface caries in experimental animals, indicating that, they may have benefit as an adjuvant complementary to fluoride for dental caries inhibition (Koo et al., 2010). In addition, they are present in high concentration mainly in grape seed extracts (Cuevas et al., 2011).

Numerous pharmacological actions of proanthocyanidins have been described, such as antioxidant, lowering of capillary permeability and fragility, reduction of collagen destruction, and suppression of inflammation (Cho et al., 2009).

The aim of the following review explores the evidenced medicinal effects of the flavonoid, proanthocyanidins either in pharmaceutical preparation or in cranberry, grape seeds or acacia bark. The article will concentrate solely on clinical trials on human beings.

\section{Dry skin problems}

The existence and advancement of dry skin minimizes defence versus foreign microbes and allergens (Fallon et al., 2009, Moniaga et al., 2010 and Oyoshib et al., 2009). Aggravation of dry skin symptoms including skin redness, itching, and edema will result in elevated serum immunoglobulin E (IgE) concentrations (Moniaga et al., 2010 and Oyoshib et al., 2009). The proof has elucidated that itching impedes with human sleep, work, and daily activities and hence lessen the life standard. Furthermore, the reduction in the life standard raised by chronic itching equals that resulted from chronic pain (Kini et al., 2011). The anti-inflammatory and skin hydration characteristic of a dietary complement and 2 topical preparation (Anthogenol) consists of oligomeric proanthocyanidins were examined. In a randomized study conducted on 42 individuals allocated in 2 groups, dietary complement (100 mg/day) and placebo. One month later, erythema was induced utilizing UV radiation. The topical formula was applied. Erythema was estimated for up to 3 days post-induction. One and two weeks thereafter, skin hydration was also examined. Both local preparations significantly reduced erythema formation and the dietary complement produced a further little intense reduction. Furthermore, both local preparations ameliorated skin hydration and the hydration was elevated in the subjects administered the dietary complement (Hughes et al ., 2007).

Skin aging is a physiological process that is gradually produced as people age. Skin aging is divided into two types, internal and external. Skin disability is controlled by internal, hormonal or environmental factors such as smoking, drinking and exposure to sunlight. Studies have shown that oxygen free radicals play a central role in the skin aging process (Bogdan and Baumann, 2008, Landau, 2007, Barbosa et al., 2010 and Naylor et al ., 2011). Another study aimed to investigate the effect of marine protein tablets and grape seeds extract on the improvement of skin deficits. The trial involved 47 male volunteers (30-45 years). They were phototypes I-IV on the Fitzpatrick scale. Volunteers have given 2 tablets of the marine supplement for 6 months. The supplement ameliorated skin erythema, hydration, $\mathrm{pH}$, and total appearance (Costa et al., 2015).

\section{Urinary tract infection (UTIs)}

In the study aimed to estimate if cranberries proanthocyanidins are capable of prohibited postoperative UTIs in individuals perform pelvic operation and undergone transurethral catheterization. Two hundred and fifty-five women performing pelvic operation were randomly allocated into two groups, one dose cranberry proanthocyanidins group $(n=132,36 \mathrm{mg} /$ day $)$ and placebo group $(\mathrm{n}=123)$. The duration of the study was 10 days. The fundamental outcome was after surgery, the urine bacterial count was estimated after 15 and 40 days. Pretreatment of cranberry proanthocyanidins did not decrease the occurrence of UTIs (Letouzey et al., 2017) .

A study aimed to investigate the effect of an oral product of extremely standardized cranberry extracts (Anthocyanin $囚$ ) in young individuals (12 -18 years They have already been diagnosed with recurrent UTIs. Thirty-six subjects were divided into two groups, standard management group $(n=17)$ and standard management combined with Anthocyanin ${ }^{\circledR}(\mathrm{n}=19)$. Treatment continued for 2 months. The number of UTIs were estimated. In addition to the number of symptom-free individuals throughout the study interval. The average number of UTIs determined through the study in the Anthocyanin ${ }^{\circledR}$ group was significantly decreased compared to the control group. Furthermore, $63.1 \%$ of Anthocyanin ${ }^{\circledR}$ treated individuals were symptom-free during the study time compared to $23.5 \%$ of control individuals (Ledda et al., 2017) .

Another study estimated the usefulness of an extremely concentrated cranberry juice for the protection against recurrent UTIs in uncircumcised male children. The study was a randomized, controlled trial. Fifty-five uncircumcised child were divided into 2 groups, group $1(\mathrm{n}=28)$ administered cranberry juice $(120 \mathrm{ml} / \mathrm{day})$ and group $2(n=27)$ administered a placebo juice. The circumcised children constitute group $3(n=12)$ administered a placebo juice likewise. The duration of the study was 6 months. The time to appearance of UTI symptoms and pyuria) was the primary outcome. Concentrated cranberry juice significantly reduced the incidence of UTI 
attacks in uncircumcised children and may exert valuable action versus Gram-negative bacteria. In addition, compared to the circumcision procedure, concentrated cranberry juice exerted higher the prophylactic action against UTI (Wan et al., 2016).

A study aimed to assess the prophylactic action of Oximacro, a cranberry extract contains a large concentration of proanthocyanidins -A, to hinder UTIs. Subjects of both sexes were included in this work. They were equally divided into a female group (19-51 years) and a male group (more than 51 years). They were both administered Oximacro (36 mg proanthocyanidins -A) twice daily for one week. The control group was administered a placebo capsule. A dose of $112 \mathrm{mg}$ Oximacro containing $36 \mathrm{mg}$ proanthocyanidins-A was found to be efficient in prohibiting UTIs when administered two times daily for one week (Occhipinti et al., 2016) .

A work investigated if the entire cranberry fruit powder (proanthocyanidin composition $=0.56 \%$ ) could hinder frequent UTIs. One hundred eighty-two ladies diagnosed to faced 2 or more UTIs attacks one year ago. Eighty-nine women were randomly included in a cranberry group $(500 \mathrm{mg}$ ) while ninety-three women were included in a control group. The treatment period was 24 weeks. The number of UTIs detected was calculated. Cranberry significantly reduced the number of UTIs. In addition, the Kaplan-Meier survival analysis displayed that the cranberry prolonged the time to the first UTIs (Vostalova et al., 2015).

A prospective study matched UTIs rate between 62 individuals subjected to double $\mathrm{J}$ catheter. Patients were equally classified into the intervention group (American cranberry, $120 \mathrm{mg}+$ standard prophylactic regimen) and the control group (standard prophylactic regimen). The study results revealed that American cranberry could be used as an adjuvant therapy to protect against UTIs in individuals utilizing double J catheter post operations (Barnoiu et al., 2015).

In a work aimed to estimate the possible protective role of cranberry extracts against UTIs in multiple sclerosis (MS) patients. The study included 171 multiple sclerosis patients. The patients were randomly included to either cranberry extracts group ( $36 \mathrm{mg} / \mathrm{day}$ ) or control group. The duration of the study was 1 year. The time to the first UTIs episode was calculated. Administering cranberry extracts did not hinder UTIs event in MS patients (Gallien et al., 2014).

In a study evaluated the recurrence rate of UTIs in patients with multiple relapses after utilizing cranberry juice. Patients (20-79 years) were randomly enrolled into 2 groups, cranberry juice group (125 ml/day) and control group (placebo juice $125 \mathrm{ml} /$ day). The duration of the study was 6 months. The primary endpoint was the recurrence of UTIs. The study results showed that cranberry juice inhibited the recurrence of UTIs among a group of the study population (Takahashi et al., 2013).

In a work aimed to evaluate the activity of cranberry juice for the inhibition of children UTIs. Forty children were included, and they were either administered cranberry juice with concentrated proantho or cranberry juice without pronto. the study continued for 1 year. The study goal was a $30 \%$ reduction in the average of UTIs. The results showed that concentrated cranberry resulted in a $65 \%$ reduction in the episodes of UTIs (Afshar et al., 2012).

In a placebo-controlled study which desired to examine the impact of cranberry formulation for the protection against UTIs in spinal cord disordered subjects. Patients with spinal cord trauma and diagnosed with neurogenic bladder were classified to take 6 months of cranberry extract formulation or placebo. The aim of this study was to assess the rate of UTIs. The results showed that cranberry formulation significantly decreased the probability of UTIs and signs. Among the cranberry treatment, 6 patients suffered 7 UTIs, compared to 16 patients and 21 UTIs in the placebo treatment. Cranberry extract formulation could be a beneficial alternative in prophylaxis against UTIs in spinal cord trauma individuals with neurogenic bladder (Hess et al., 2008).

\section{Gingivitis}

Gingivitis is primarily inflammation of the periodontal gland tissue, which when developed, is accompanied by a bacterial infection that destroys the supporting tissues of the teeth and gums (Bosshardt and Selvig, 1997). Despite, in vitro trials published about the interaction between the proanthocyanidins and bacterial infection of the dental tissue have concluded extremely perfect outcomes, there are no data with the convenient design that evaluate the clinical activity of oral proanthocyanidins on the dental tissue inflammation in vivo (Väänänen et al., 1993, Govindaraj et al., 2010 and Löhr et al., 2011) . A prospective, double-blind, randomized, controlled clinical trial which aimed to assess the therapeutic potential of oral proanthocyanidins on experimentally induced gingivitis. The study follows up period was 21 days. Twenty subjects were divided into 2 groups, an experimental group, and a control group. The estimated parameters were as follows, the Silness and Löe index, the dental bleeding index, the plaque index, interleukin six, and the changes in the glitter of the gingiva. No other hygiene protocols were followed in the study period. Proanthocyanidins produced a very good impact on periodontal tissues health. however, no actions on the formation of plaque were observed (BoronatCatalá et al., 2014, Morelli et al., 2014, Syndergaard et al., 2014 and Gümüş et al., 2015 and Díaz Sánchez et al ., 2017).

A control and a randomized clinical study aimed at assessing the clinical and microbiological outcomes of proanthocyanidins and secnidazole on gingivitis. Seventy-five individuals with chronic gingivitis were classified into three equal groups, secnidazole, proanthocyanidins, and placebo control. The study period was 3 months of 
treatment. Plaque index (PI), gingival index (GI), gingival bleeding index (GBI), probing pocket depth (PPD), clinical attachment level (CAL), and microbial count were determined at zero time and at the end of the study period. The data displayed that the two intervention groups significantly decreased GBI, GI, and PPD compared to the control group. However, there were no significant differences in PI and CAL. Also, in both treatment groups, there was a significant reduction in the bacterial counts. The study results concluded that the utilization of either proanthocyanidins or secnidazole together with scaling and root planning ameliorates gingivitis as they are potent in decreasing the oral microbes and fulfil markedly superior clinical outcomes (Li et al., 2017).

\section{Menopausal symptoms}

A randomized study objective to evaluate the impact of administering proanthocyanidins seed extracts on menopausal signs, body composition, and blood pressure in middle-aged female subjects. Ninety-six ladies were divided into 3 groups, proanthocyanidins (100 mg daily), proanthocyanidins ( $200 \mathrm{mg}$ daily), and placebo. The study period was 8 weeks. Grape seeds proanthocyanidins extract significantly ameliorated menopausal symptoms and hot flash scores. In addition, proanthocyanidins significantly decreased both insomnia and anxiety scores. The extract improves the body lean mass and muscle mass. Grape seeds proanthocyanidins extract significantly reduced menopausally associated hypertension (Terauchi et al., 2014).

\section{Oxidative stress}

In a placebo-controlled, double-blind trial aimed to evaluate the impact of Vitis vinifera proanthocyanidin extract on oxidative stress measures and blood concentration of adiponectin, leptin and low-density lipoprotein after $48 \mathrm{hr}$ of heavy exercise. The study was carried out on 54 adult army cadets who were classified into 2 equal groups, control and proanthocyanidin (200 $\mathrm{mg}$ dose) once before performing heavy exercises. Proanthocyanidin significantly decreased low-density lipoprotein concentration while increasing plasma adiponectin level measured 1-day post exercises (Gonçalves et al., 2017).

\section{Wound healing}

The skin acts as a barrier to protect the body against various environmental factors, as the improper parts of it may expose man to disability and may even die. Despite numerous researches have proposed the benefits of grape seed extracts in maintaining wound healing in experimental animals, there are no trials has been conducted on the man yet. In this work, the topical influence of grape seed extracts on skin wound healing in man was examined. This study is a double-blind clinical work which utilized two groups of treatment and control. Surgery was conducted on skin trauma such as skin tags and moles that were located on the neck, trunk, and limbs (face not included). Removal of skin disorders was performed by surgical scissors. Grape seed extracts cream $2 \%$ was applied. The data proposed full wound healing observed on day 8 after pro treatment while it takes about 14 days for the control group (Hemmati et al., 2014).

\section{Leg swelling}

Leg swelling is a contemporary disorder of sedentary employed ladies. This work aimed to examine the influence of the ingestion of grape seed extract on leg edema in healthy volunteer ladies during sitting. A long sedentary status was kept for $6 \mathrm{~h}$ post grape seed extracts or placebo ingestion. Grape seed extracts significantly decreased leg volume distension and leg water, while raised the body extracellular fluid (Sano et al., 2013).

\section{Radiation injury}

This work objective was to investigate the inhibitory influence of grape procyanidins on radiation trauma in radiation-contacted subjects. The study was carried out on 60 subjects who were closely related to radiation. They were included to either procyanidins group $(100 \mathrm{mg} / \mathrm{day})$ or placebo group. Furthermore, another 15 healthy volunteers not in touch with radiation served as a normal group. The treatment protocol was continued for 2 months. Several antioxidant parameters were measured in the blood (total antioxidant capacity and malondialdehyde). In addition, the expression levels of proliferative cell nuclear antigen, Bcl-2 and Bax were determined. Procyanidins significantly increased total antioxidant capacity and proliferative cell nuclear antigen. However, procyanidins significantly decreased malondialdehyde and Bax expression. Procyanidins prevented radiation injury (Liu and Zhong, 2008).

\section{Helicobacter pylori}

This study aimed to compare the impact of cranberry juice rich in proanthocyanidins and Lactobacillus johnsonii La1 on Helicobacter pylori colonized children. The study was conducted on 295 children who showed positive (13) C-urea breath test for Helicobacter pylori. They were divided into 4 groups, cranberry juice + probiotic Lactobacillus johnsonii La1, placebo + probiotic Lactobacillus johnsonii La1, cranberry juice + heatkilled probiotic Lactobacillus johnsonii La1, and placebo juice + heat-killed probiotic Lactobacillus johnsonii La1. Cranberry juice was administered in a dose of $200 \mathrm{ml} / \mathrm{day}$ while probiotic Lactobacillus johnsonii La1 was 
administered in a dose of $80 \mathrm{ml} /$ day. Treatment was continued for 3 weeks. (13) C-urea breath test was estimated after 3 weeks and the children who showed negative (13) C-urea breath test are examined again after 4 weeks washout period. The results of this study proposed that administration of cranberry juice or probiotic Lactobacillus johnsonii alone may be helpful in the treatment of children colonized by Helicobacter pylori. Although, there were no additive repressing impacts on Helicobacter pylori colonization in the combination group (Gotteland et al., 2008) .

\section{Melasma}

Melasma, an acquired hyperpigmentation, is frequently stubborn to any therapy. This study examined the impact of proanthocyanidin on melasma reduction. Grape seed extracts contained a high amount of proanthocyanidin was given to 12 ladies with chloasma for 180 days and to 11 of these 12 for an additional 150 days. In $83 \%$ of the ladies, administration of Grape seed extracts significantly ameliorated melasma after 180 days. After the additional 150 days, only 54\% of the cases showed improvement. The ameliorative action of grape seed extracts was maximally accomplished post 6 months and there was no additional amelioration after this time. Grape seed extracts administration for 5 months may stop melasma exacerbation before to the summer season (Yamakoshi et al., 2004) .

\section{CONCLUSION}

Many human trials have disclosed prevalent medicinal application of cranberry and grape seed their active component proanthocyanidins. These researches suggested its potential implementation as medicine or food supplement for controlling numerous illness. The curative and/or protective impacts of proanthocyanidins could be referred to their demonstrated antioxidant action. This assumes the emergency for extra human trials, to evidence or disproves any pharmacological activity. These days, numerous clinical trials are in progress to search the curative ability of cranberry, grape seed and proanthocyanidins. However, prior to their usage, cranberry, grape seed, and proanthocyanidins should be compared with the currently approved drugs. Furthermore, large-scale, multicentre studies are crucial to estimate the potency of these generous food supplements.

\section{REFERENCES}

Afshar, K., L. Stothers, H. Scott and A.E. MacNeily, 2012. Cranberry juice for the prevention of pediatric urinary tract infection: A randomized controlled trial. Journal of Urology, 188(4S): 1584-1587.

Barbosa, K.B.F., N.M.B. Costa, R. de C.G. Alfenas, S.O. De Paula, V.P.R. Minim and J. Bressan, 2010. Estresse oxidativo: conceito, implicações e fatores modulatórios. Revista de Nutrição, 23(4): 629-643.

Barnoiu, O.S., J. Sequeira-García del Moral, N. Sanchez-Martínez, P. Díaz-Molina, L. Flores-Sirvent and V. Baena-González, 2015. Valor preventivo adyuvante del arándano rojo americano (proantocianidinas $120 \mathrm{mg}$ ) en las infecciones del tracto urinario tras la colocación de catéter ureteral. Actas Urológicas Españolas, 39(2): 112-117.

Bogdan A., I. and L. Baumann, 2008. Antioxidants used in skin care formulations. Skin Therapy Letter, 13(7): $5-9$.

Boronat-Catalá, M., M. Catalá-Pizarro and J. V Bagán Sebastián, 2014. Salivary and crevicular fluid interleukins in gingivitis. Journal of Clinical and Experimental Dentistry, 6(2): e175-9.

Bosshardt, D.D. and K.A. Selvig, 1997. Dental cementum: the dynamic tissue covering of the root. Periodontology 2000, 13: 41-75.

Cho, M.L., Y.J.Heo, M.K. Park, H.J. Oh, J.S. Park, Y.J. Woo, J.H. Ju, S.H. Park, H.Y. Kim and J. K. Min, 2009. Grape seed proanthocyanidin extract (GSPE) attenuates collagen-induced arthritis. Immunology Letters, 124(2): 102-110.

Costa, A., E.S. Pegas Pereira, E.C. Assumpção, F.B. Calixto Dos Santos, F.S. Ota, M. de Oliveira Pereira, M.C. Fidelis, R. Fávaro, S.S. Barros Langen, L.H. Favaro de Arruda and E.N. Abildgaard, 2015. Assessment of clinical effects and safety of an oral supplement based on marine protein, vitamin $\mathrm{C}$, grape seed extract, zinc, and tomato extract in the improvement of visible signs of skin aging in men. Clinical, Cosmetic and Investigational Dermatology, 8: 319-28.

Cuevas, V.M., Y.R. Calzado, Y.P. Guerra, A.O. Yera, S.J. Despaigne, R.M. Ferreiro and D.C. Quintana, 2011. Effects of grape seed extract, vitamin $\mathrm{C}$, and vitamin $\mathrm{E}$ on ethanol- and aspirin-induced ulcers. Advances in Pharmacological Sciences, 2011: 740687.

De la Iglesia, R., F.I. Milagro, J. Campión, N. Boqué and J.A. Martínez, 2010. Healthy properties of proanthocyanidins. Bio Factors, 36(3): 159-168.

Díaz Sánchez, R.M., G. Castillo-Dalí, A. Fernández-Olavarría, R. Mosquera-Pérez, J.M. Delgado-Muñoz, J.L. Gutiérrez-Pérez and D. Torres-Lagares, 2017. A Prospective, double-blind, randomized, controlled clinical 
trial in the gingivitis prevention with an oligomeric proanthocyanidin nutritional supplement. Mediators of Inflammation, 2017: 1-7.

Dong, C., 2014. Protective effect of proanthocyanidins in cadmium induced neurotoxicity in mice. Drug Research, 65(10): 555-560.

Fallon, P.G., T. Sasaki, A. Sandilands, L.E. Campbell, S.P. Saunders, N.E. Mangan, J.J. Callanan, H. Kawasaki, A. Shiohama, A. Kubo, J.P. Sundberg, R.B. Presland, P. Fleckman, N. Shimizu, J. Kudoh, A.D. Irvine, M. Amagai and W.H.I. McLean, 2009. A homozygous frameshift mutation in the mouse Flg gene facilitates enhanced percutaneous allergen priming. Nature Genetics, 41(5): 602-608.

Foo,L.Y., Y. Lu, A.B. Howell and N. Vorsa, 2000. The structure of cranberry proanthocyanidins which inhibit adherence of uropathogenic P-fimbriated Escherichia coli in vitro. Phytochemistry, 54(2): 173-81.

Gallien, P., G. Amarenco, N. Benoit, V. Bonniaud, C. Donzé, J. Kerdraon, M. de Seze, P. Denys, A. Renault, F. Naudet and J.M. Reymann, 2014. Cranberry versus placebo in the prevention of urinary infections in multiple sclerosis: a multicenter, randomized, placebo-controlled, double-blind trial. Multiple Sclerosis Journal, 20(9): 1252-1259.

Gonçalves, M.C., M.C.F. Passos, C.F. de Oliveira, J.B. Daleprane and J.C. Koury, 2017. Effects of proanthocyanidin on oxidative stress biomarkers and adipokines in army cadets: a placebo-controlled, double-blind study. European Journal of Nutrition, 56(2): 893-900.

Gotteland, M., M. Andrews, M. Toledo, L. Muñoz, P. Caceres, A. Anziani, E. Wittig, H. Speisky and G. Salazar, 2008. Modulation of Helicobacter pylori colonization with cranberry juice and Lactobacillus johnsonii La1 in children. Nutrition, 24(5): 421-426.

Govindaraj, J., P. Emmadi, Deepalakshmi, V. Rajaram, G. Prakash and R. Puvanakrishnan, 2010. Protective effect of proanthocyanidins on endotoxin induced experimental periodontitis in rats. Indian Journal of Experimental Biology, 48(2): 133-42.

Gümüş, P., Ö. Özçaka, B. Ceyhan-Öztürk, A. Akcali, D.F. Lappin and N. Buduneli, 2015. Evaluation of biochemical parameters and local and systemic levels of Osteoactive and B-cell stimulatory factors in gestational diabetes in the presence or absence of gingivitis. Journal of Periodontology, 86(3): 387-397.

Hemmati, A.A., M. Foroozan, G. Houshmand, Z.B. Moosavi, M. Bahadoram and N.S. Maram, 2014. The topical effect of grape seed extract $2 \%$ cream on surgery wound healing. Global Journal of Health Science, 7(3): $52-8$.

Hess, M.J., P.E. Hess, M.R. Sullivan, M. Nee and S. V Yalla, 2008. Evaluation of cranberry tablets for the prevention of urinary tract infections in spinal cord injured patients with neurogenic bladder. Spinal Cord, 46(9): 622-626.

Howell, A.B., J.D. Reed, C.G. Krueger, R. Winterbottom, D.G. Cunningham and M. Leahy, 2005. A-type cranberry proanthocyanidins and uropathogenic bacterial anti-adhesion activity. Phytochemistry, 66(18): 2281-2291.

Hughes-Formella, B., O. Wunderlich and R. Williams, 2007. Anti-Inflammatory and skin-hydrating properties of a dietary supplement and topical formulations containing oligomeric proanthocyanidins. Skin Pharmacology and Physiology, 20(1): 43-49.

Kini, S.P., L.K. DeLong, E. Veledar, A.M. McKenzie-Brown, M. Schaufele and S.C. Chen, 2011. The impact of pruritus on quality of life. Archives of Dermatology, 147(10): 1153.

Koo, H., S. Duarte, R.M. Murata, K. Scott-Anne, S. Gregoire, G.E. Watson, A.P. Singh and N. Vorsa, 2010. Influence of cranberry proanthocyanidins on formation of biofilms by Streptococcus mutans on Salivacoated apatitic surface and on dental caries development in vivo. Caries Research, 44(2): 116-126.

Krenn, L., M. Steitz, C. Schlicht, H. Kurth and F. Gaedcke, 2007. Anthocyanin- and proanthocyanidin-rich extracts of berries in food supplements--analysis with problems. Die Pharmazie, 62(11): 803-12.

Landau, M., 2007. Exogenous factors in skin aging, In Curr. Probl. Dermatol., pp: 1-13.

Ledda, A., G. Belcaro, M. Dugall, A. Riva, S. Togni, R. Eggenhoffner and L. Giacomelli, 2017. Highly standardized cranberry extract supplementation (Anthocran ${ }^{\circledR}$ ) as prophylaxis in young healthy subjects with recurrent urinary tract infections. European Review for Medical and Pharmacological Sciences, 21(2): 389-393.

Letouzey, V., D. Ulrich, C. Demattei, S. Alonso, S. Huberlant, J.-P. Lavigne and R. de Tayrac, 2017. Cranberry capsules to prevent nosocomial urinary tract bacteriuria after pelvic surgery: a randomised controlled trial. BJOG: An International Journal of Obstetrics \& Gynaecology, 124(6): 912-917.

Li, M., R. Li, Q. Jin, J. Pang and Z. Xu, 2017. The efficacy of proanthocyanidins and secnidazole in the treatment of chronic periodontitis after scaling and root planing therapy. Journal of Biological Regulators and Homeostatic Agents, 31(1): 93-97.

Liu, J. and J. Zhong, 2008. [Study on protective effect of grape procyanidins in radiation injury in radiationcontacted persons]. Zhonghua Yu Fang Yi Xue Za Zhi [Chinese Journal of Preventive Medicine], 42(4): 264-7. 
Löhr, G., T. Beikler, A. Podbielski, K. Standar, S. Redanz and A. Hensel, 2011. Polyphenols from Myrothamnus flabellifolia Welw. inhibit in vitro adhesion of Porphyromonas gingivalis and exert anti-inflammatory cytoprotective effects in KB cells. Journal of Clinical Periodontology, 38(5): 457-469.

Moniaga, C.S., G. Egawa, H. Kawasaki, M. Hara-Chikuma, T. Honda, H. Tanizaki, S. Nakajima, A. Otsuka, H. Matsuoka, A. Kubo, J. Sakabe, Y. Tokura, Y. Miyachi, M. Amagai and K. Kabashima, 2010. Flaky tail mouse denotes human atopic dermatitis in the steady state and by topical application with Dermatophagoides pteronyssinus extract. The American Journal of Pathology, 176(5): 2385-93.

Morelli, T., M. Stella, S.P. Barros, J.T. Marchesan, K.L. Moss, S.J. Kim, N. Yu, M.B. Aspiras, M. Ward and S. Offenbacher, 2014. Salivary biomarkers in a biofilm overgrowth model. Journal of Periodontology, 85(12): 1770-8.

Naylor, E.C., R.E.B. Watson and M.J. Sherratt, 2011. Molecular aspects of skin ageing. Maturitas, 69(3): 249256.

Nicolosi, D., G. Tempera, C. Genovese and P.M. Furneri, 2014. Anti-adhesion activity of A2-type proanthocyanidins (a cranberry major component) on uropathogenic E. coli and P. mirabilis Strains. Antibiotics (Basel, Switzerland), 3(2): 143-54.

Occhipinti, A., A. Germano and M.E. Maffei, 2016. Prevention of urinary tract infection with Oximacro, A cranberry extract with a high content of A-type proanthocyanidins: A Pre-Clinical Double-Blind Controlled Study. Urology Journal, 13(2): 2640-9.

Oyoshi, M.K., G.F. Murphy and R.S. Geha, 2009. Filaggrin-deficient mice exhibit TH17-dominated skin inflammation and permissiveness to epicutaneous sensitization with protein antigen. Journal of Allergy and Clinical Immunology, 124(3): 485-493.e1.

Pan, S.-Y., S.-F. Zhou, S.-H. Gao, Z.-L. Yu, S.-F. Zhang, M.-K. Tang, J.-N. Sun, D.-L. Ma, Y.-F. Han, W.-F. Fong and K.-M. Ko, 2013. New Perspectives on how to discover drugs from herbal medicines: CAM's outstanding contribution to modern therapeutics. Evidence-Based Complementary and Alternative Medicine : ECAM, 2013: 627375.

Ravishankar, D., A.K. Rajora, F. Greco and H.M.I. Osborn, 2013. Flavonoids as prospective compounds for anti-cancer therapy. The International Journal of Biochemistry \& Cell Biology, 45(12): 2821-2831.

Sano, A., S. Tokutake and A. Seo, 2013. Proanthocyanidin-rich grape seed extract reduces leg swelling in healthy women during prolonged sitting. Journal of the Science of Food and Agriculture, 93(3): 457-462.

Singh, A.P., T. Wilson, A.J. Kalk, J. Cheong and N. Vorsa, 2009. Isolation of specific cranberry flavonoids for biological activity assessment. Food Chemistry, 116(4): 963-968.

Syndergaard, B., M. Al-Sabbagh, R.J. Kryscio, J. Xi, X. Ding, J.L. Ebersole and C.S. Miller, 2014. Salivary biomarkers associated with gingivitis and response to therapy. Journal of Periodontology, 85(8): e295e303.

Takahashi, S., S. Takahashi, R. Hamasuna, M. Yasuda, S. Arakawa, K. Tanaka, K. Ishikawa, H. Hayami, S. Yamamoto, T. Kubo, T. Matsumoto, R. Hamasuna, T. Matsumoto, M. Yasuda, S. Arakawa, K. Tanaka, K. Ishikawa, H. Kiyota, H. Hayami, S. Yamamoto and T. Kubo, 2013. A randomized clinical trial to evaluate the preventive effect of cranberry juice (UR65) for patients with recurrent urinary tract infection. Journal of Infection and Chemotherapy, 19(1): 112-117.

Terauchi, M., N. Horiguchi, A. Kajiyama, M. Akiyoshi, Y. Owa, K. Kato and T. Kubota, 2014. Effects of grape seed proanthocyanidin extract on menopausal symptoms, body composition, and cardiovascular parameters in middle-aged women: a randomized, double-blind, placebo-controlled pilot study. Menopause (New York, N.Y.), 21(9): 990-6.

Väänänen, M.K., H.A. Markkanen, V.J. Tuovinen, A.M. Kullaa, A.M. Karinpää and E.A. Kumpusalo, 1993. Periodontal health related to plasma ascorbic acid. Proceedings of the Finnish Dental Society. Suomen Hammaslaakariseuran Toimituksia, 89(1-2): 51-9.

Vostalova, J., A. Vidlar, V. Simanek, A. Galandakova, P. Kosina, J. Vacek, J. Vrbkova, B.F. Zimmermann, J. Ulrichova and V. Student, 2015. Are high proanthocyanidins key to cranberry efficacy in the prevention of recurrent urinary tract infection?. Phytotherapy Research, 29(10): 1559-1567.

Wan, K.S., C.K. Liu, W.K. Lee, M. C. Ko and C.S. Huang, 2016. Cranberries for preventing recurrent urinary tract infections in uncircumcised boys. Alternative Therapies in Health and Medicine, 22(6): 20-23.

Yamakoshi, J., A. Sano, S. Tokutake, M. Saito, M. Kikuchi, Y. Kubota, Y. Kawachi and F. Otsuka, 2004. Oral intake of proanthocyanidin-rich extract from grape seeds improves chloasma. Phytotherapy Research, 18(11): 895-899.

Yuan, H., Q. Ma, L. Ye and G. Piao, 2016. The traditional medicine and modern medicine from natural products. Molecules, 21(5): 559. 